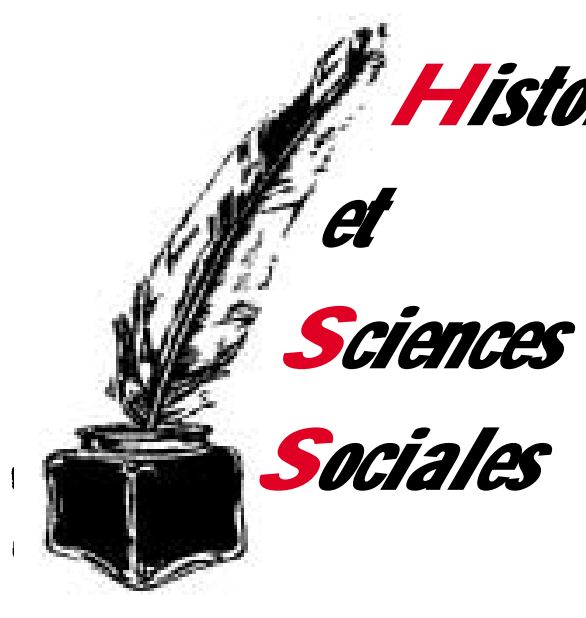

\title{
Des mouches et des hommes, dans un laboratoire japonais de génétique du comportement
}

L'anthropologie des sciences offre, sur les pratiques et les én on cés scientifiques, un point de vue qui permet de resituer les uns comme les autres dans leur contexte social et culturel d'élaboration. L'ethnologie d'un laboratoire japonais de gén étique du comportement permet d'appréhen der l'articulation en tre les dimensions locale (celle d'un laboratoire installé au Japon) et globale (celle de la communauté

\begin{abstract}
scientifique internationale). A travers l'analyse de certains des processus par lesquels les chercheurs font venir à l'existence les faits scientifiques, le laboratoire japonais apparaît comme un lieu dans lequel des individus disposent de véritables moyens (d'expression, d'action) pour étendre à l'extérieur (faire naître et propager) de nouvelles configurations culturelles.
\end{abstract}

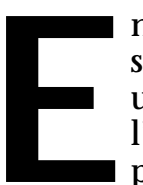
n mai 1999 se tient, à la Maison de la culture du Japon, une conférence donnée par I'éminent orateur Leo Esaki, physicien japonais, Prix Nobel de physique en 1973. A peine l'incontournable cycle des politesses a-t-il été achevé que Esaki ouvre ainsi son discours: "Science is a sort of international culture». A la «culture traditionnelle» qu'Esaki définit comme «conservatrice», «tournée vers I'histoire» et «attachée aux récits régionaux du passé», s'oppose la «culture de la science», «progressiste», «tournée vers le futur», et «globale». L'une et l'autre cultures semblent ainsi irrémédiablement contraintes à suivre des voies différentes. En matière de culture, donne à penser Esaki, il faut choisir: choisir la permanence ou bien le progrès; la tradition ou la science. Le Japon est bien placé pour le savoir, lui qui, aux dires de Esaki, a fait le choix de sacrifier la première à la seconde, et participer ainsi au tourbillon de la mondialisation. Et sicien sur ce terrain et constater avec lui la modernité de son pays: ne peut-on pas reconnaître en ce dernier tous les signes caractéristiques de cette modernité qui est aussi la nôtre ? Une économie performante, un système démocratique, une technologie de pointe ne finissent-ils pas de mettre le Japon à parité avec les nations occidentales?

En raisonnant de la sorte, Esaki remet à I'honneur, sans le savoir, une tradition bien connue parmi les anthropologues, et qui porte même un nom: le Grand Partage. Cette tradition, qui consiste à stipuler "l'existence d'une ligne univoque de séparation entre deux genres et deux seulement de sociétés et de cultures: sociétés primitives et sociétés civilisées, sociétés simples et sociétés complexes, sociétés tradition nelles et sociétés modernes» [1], a placé les systèmes cognitifs et de représentation du monde suivant deux ordres incommensurables. Que I'on se situe de part ou d'autre de la ligne démarcative, et l'on a, selon les terminologies propres aux différents anthropologues, d'un côté les savoirs religieux et de l'autre le savoir scientifique, d'un côté un esprit pré-scientifique et de l'autre l'esprit scientifique, d'un côté le raisonnement quotidien et de l'autre le raisonnement scientifique, d'un côté le bricolage et de I'autre la science [2] ${ }^{1}$. La tradition se voit opposée à la rationalité, les valeurs culturelles (dépendantes du contexte) aux valeurs universelles (fondamentalement autonomes). L'effet d'un tel découpage a eu, sur la méthodologie, des répercussions indéniables: tandis que les systèmes cognitifs des sociétés non occidentales étaient étudiés, par cette sous-discipline de l'anthropologie appelée l'ethnoscience, dans leur contexte culturel d'élaboration et d'expression, le système cognitif occidental moderne (le plus répandu, faudrait-il dire: la science) constituait la principauté de l'épistémologie, cette «science des

${ }^{1}$ Cette dernière opposition est particulièrement célèbre dans le champ del'anthropologie puisqu'on la doit à l'un des pères de l'anthropologie française, Claude Lévi-Strauss [2]. 
sciences» [3] qui laissait globalement pour compte le contexte culturel. L'on pourrait figurer ainsi le Grand Partage:

Dans la première bulle, inclusive, les systèmes cognitifs, qui épuisent la réalité naturelle et l'ordonnent, sont expliqués par la société; dans la seconde, en revanche, exclusive cellelà, les systèmes, visant pourtant les mêmes fins, sont expliqués par la nature seule. Les premiers peuvent faire l'objet d'une analyse anthropologique tandis que l'analyse des seconds revient tout entière aux spécialistes des sciences dites dures, ou aux épistémologues qui, convenonsen, produisent souvent à leur endroit un «discours redondant et publicitaire» [3] (figure 1).

Non que certains épistémologues (Thomas Kuhn le premier, ou du moins le plus célèbre) n'aient conçu un modèle explicatif qui use bel et bien du concept de culture (voir en particulier [4]). Cependant, il s'agit alors de la culture locale, celle d'une spécialité scientifique qui se reconnaîtrait sous un même paradigme. Les sociologues des sciences après eux ont ouvert les laboratoires aux investigations sociologiques en tenant de front l'analyse des communautés scientifiques et celle des contenus scientifiques, et en soumettant les unes comme les autres aux mêmes appareillages méthodologiques et conceptuels. Les ethnographies pionnières de laboratoire scientifique remontent au début des années 1970: Michael Lynch, Sharon Traweek et Bruno Latour entament en même temps, mais dans des endroits différents, des analyses détaillées des pratiques scientifiques [ 58]; ils décrivent les procédures motrices de l'activité scientifique comme étant en même temps techniques, politiques, sociales. Avec de telles études, le concept de culture gagne en densité: la culture locale ne sert plus seulement à qualifier un groupement humain, celui d'un laboratoire ou bien d'une communauté de chercheurs; elle rend compte également des énoncés scientifiques qui apparaissent dès lors comme des "éléments culturels», des faits scientifiques qui deviennent des «produc-

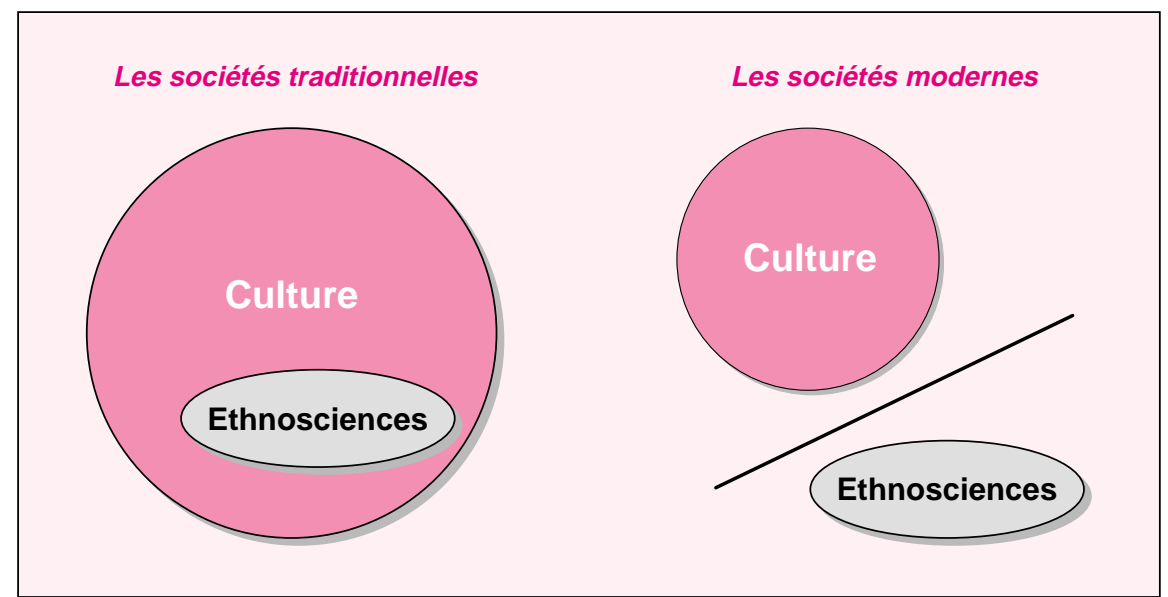

Figure 1. Le grand partage.

tions culturelles locales», ou encore des outils scientifiques conçus comme des «éléments d'une culture locale». La science se fait alors aussi diverse que ses pratiques et que ses promoteurs: les sciences constituent « un ensemble de pratiques culturelles [ et] ce qui retient l'attention, ce sont les pratiques scientifiques locales et contingentes. Le travail scientifique est alors montré comme lié à des éléments culturels multiples (savoirs tacites incorporés dans les individus, compétences, instruments, règles) » [9].

Reste alors à articuler cet usage particulier du concept de culture scientifique avec celui de culture au sens anthropologique du terme. C'est ce que font, de manière on ne peut plus convaincante, Steven Shapin et Simon Schaffer en étudiant la mise en place de notre cosmologie moderne [10]. Retraçant les termes de la controverse qui opposa, dans la seconde moitié du Xvile siècle, Thomas Hobbes et Robert Boyle à propos de la capacité de la pompe à air à produire du vide, les deux auteurs montrent comment, depuis Boyle, les laboratoires scientifiques sont tenus pour des lieux en dehors de la culture et tournés vers la mise en ordre de la nature. Dans cette configuration, la nature apparaît comme une et ordonnée tandis que la culture est complexe et ne se laisse pas réduire à un ensemble de lois. C'est en vertu de cette séparation respectée qu'on tient les scientifiques à même de pro-

duire un discours vrai et légitime pour tout dire, universel. En explicitant ainsi le contexte culturel et social qui vit la naissance des laboratoires scientifiques, le travail de Shapin et Schaffer, quoique historique, fondait la possibilité d'une véritable anthropologie des sciences. Dans un contexte contemporain, l'ethnologie d'un laboratoire de biologie japonais ${ }^{2}$ permet de comprendre comment s'articulent l'appartenance à la société japonaise et l'appartenance à la communauté scientifique internationale, dont le fonctionnement est - se plaît-on à penser - largement indépendant de normes culturelles ou sociales. Situé dans la banlieue de Tôkyô, le laboratoire étudié ${ }^{3}$, le Y. Behavior's genes Project, a vu le jour en 1994 grâce au programme gouvernemental de recherche fondamentale ERATO (Exploratory Research for Advanced Science and Technology). Ce programme fut explicitement conçu pour répondre aux critiques venues de l'Occident, critiques ressassées et diversement énoncées suivant lesquelles les scientifiques japonais auraient un sens restreint de l'innovation et un sens aigu de l'imitation. Le but d'ERATO est de contrer ce qui est identifié comme faisant obstacle à l'innovation japonaise, à savoir :

\footnotetext{
${ }^{2}$ Cette ethnologie a fait l'objet d'un travail de thèse, soutenu en mai 2000 à l'université Paris X-N anterre: «Et le scientifique tint le monde».

3 J'y ai mené une étude de mars 1996 à juillet 1997.
} 
une organisation hiérarchique forte, un manque d'interdisciplinarité et de flexibilité. Le laboratoire est donc à lui seul un lieu d'élaboration d'identités tant politiques qu'individuelles: en lui, le Japon se repositionne par rapport au reste du monde; en lui, la spécificité japonaise, tellement prisée [11], trouve un nouveau support. Ce double aspect apparaît de manière flagrante dans les liens que tisse le laboratoire japonais avec d'autres laboratoires de par le monde - liens qui montrent avec force la tension qui leur est inhérente, entre une dimension locale (le Japon qui se pense isolé aux confins de l'Orient) et une dimension globale (la communauté scientifique internationale, américaine en particulier, conçue comme soudée et représentative).

La science a beau être internationale, il est ainsi troublant de constater combien parler d'elle au Japon conduit d'emblée sur le terrain houleux de l'altérité. En 1996, dans ce laboratoire de génétique du comportement des drosophiles, il n'est guère de moment où les problèmes - même scientifiques, même techniques - ne se posent en référence à cet autre, occidental, américain, dont on s'est évertué à gagner le respect et qui continue à faire ombrage. La pratique scientifique y apparaît très vite indissociable de la conscience aiguë que l'on a de son extériorité. On sait, au Japon, que la science vient d'ailleurs; on sait dans quelles circonstances, avec quelles contraintes, elle fut introduite (pour une histoire des sciences au Japon, voir [12]). L es études tant japonaises qu'occidentales qui se sont intéressées à l'histoire de son développement sont là pour rappeler à l'envi sa particularité. La dichotomie est ici un recours rhétorique facile qui fige les termes de la comparaison: le Japon, dans son histoire scientifique et technique, s'opposerait à I'O ccident comme «l'élève» s'opposerait au «maître», le «copieur» au «copié» ou la «victime» à «l'agres-

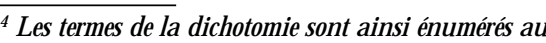
fil des pages dans l'analyse de F.L. Low. seur » [13] ${ }^{4}$. Dans le premier cas, le développement scientifico-technique serait mû par un «processus extérieur » tandis que dans le second, il naîtrait d'un «processus naturel interne» [14]. Finalement, l'introduction de la science au Japon n'est pas de I'histoire tellement ancienne que les chercheurs, tant japonais qu'étrangers, n'aient l'occasion d'y faire référence dans des situations bien contemporaines, bien internationales. Tout le monde s'y met, donc. La science, certes, n'a pas de frontières mais dans l'agrégat international, le Japon reste une île séparée du continent et éprouve chaque jour l'amertume de son isolement. Au sein du laboratoire japonais, chaque écriture d'article, chaque présentation à un colloque, chaque collaboration, chaque expérimentation - jusqu'aux drosophiles ellesmêmes et aux séquences d'ADN ne font que titiller et titiller encore un certain sens de l'altérité.

Les chercheurs du laboratoire étudié travaillent sur les mécanismes génétiques qui contrôlent le comportement sexuel des drosophiles. Nous allons voir ici comment la recherche sur cet objet insolite participe de ces processus identitaires. Plus précisément, je me propose ici de considérer la dialectique entre les dimensions locale et globale, à l'œuvre dans les processus qui visent à doter les drosophiles d'un nom et d'une identité.

\section{De la nature dans le laboratoire: se libérer du sens commun}

La nature de laboratoire a d'abord quelque chose de déroutant pour un anthropologue et, de fait, le regard naïf qu'il porte sur elle est propre à lui ôter toute évidence. Lorsque, à mon arrivée dans le laboratoire japonais, je me mets en quête de la drosophile, j'ai bien évidemment une idée de ce à quoi elle ressemble. Pour l'avoir rencontrée dans d'autres laboratoires, en d'autres lieux, je sais qu'elle est petite - ce qui la distingue clairement de la grosse mouche bleue familière à chacun et I'apparente davantage au moucheron auquel on ne prête nulle attention tant sa présence est insignifiante. Je m'at- tends donc à la rencontrer dès les portes du laboratoire franchies. Or j'en entends parler, je lis des articles parus à son sujet, je la vois décapitée, observée, mesurée, teintée, broyée; je la vois en partie (les neurones, les yeux, etc.) ou entière mais toujours dans des situations qui me laissent perplexes pour être trop éloignées du fameux sens commun. II me faut un certain temps avant d'envisager que la drosophile n'est peut-être, après tout, que l'ensemble de ces morceaux épars, disséminés dans les multiples salles du laboratoire. Ce constat dressé, le laboratoire m'apparaît différemment: il ne constitue pas un lieu dans lequel la nature est découverte, mais un espace, générique, qui modifie l'identité des objets naturels et leur en confère de nouvelles. En étant ainsi apprivoisés [15] $]^{5}$ par un collectif implanté localement, ces objets doivent être littéralement tenus pour des produits du laboratoire. C'est ainsi d'une nature qu'il s'agit, et non de la nature.

Les apparences sont donc trompeuses - car c'est bien d'abord en des termes similaires qu'on parle de la drosophile d'un bout à l'autre de la planète Science. Que I'on se trouve aux États-Unis, en France ou bien au Japon, on loue chez elle les mêmes qualités - dont on saura, semble-t-il, d'autant mieux apprécier la valeur qu'elles seront mises bout à bout: piochées au hasard de la littérature drosophiliste, les introductions des articles, qui décrivent certains aspects de l'insecte, égrènent ainsi à loisir ces qualités, sans souci majeur de leur ordre, tant elles contribuent, toutes ensembles et à même niveau, à faire de la mouche de laboratoire un organisme exemplaire. Taille de l'insecte, cycle de

\footnotetext{
5 Karin Knorr-Cetina décrit ainsi ce mouvement d'appropriation: "Laboratory sciences bring objects "home" and manipulate them "on their own terms" in the laboratory. (...) Laboratories allow for some kind of «homing in » of natural processes; the processes are "brought home" and made subject only to the local conditions of the social order. The power of the laboratory (but of course also its restrictions) resides precisely in its exclusion of nature as it is independent of laboratories and in its «enculturation» of natural objects» [15].
} 
vie, taille du génome, centaines de généticiens qui ont su, durant près d'un siècle, profiter des avantages inhérents à la drosophile, font décidément d'elle un insecte formidable $[16]^{6}$. Ces qualités sont à ce point efficaces qu'elles permettent des regroupements humains qui défient les frontières: les drosophilistes semblent avoir, nationalement ou internationalement, des choses à se dire et à partager sur la seule base de l'organisme expérimental sur lequel ils travaillent. Et c'est ainsi que, régulièrement, des chercheurs se retrouvent ou s'assemblent, qui sous l'égide des drosophilistes de France, qui lors d'un quelconque séminaire local Drosophilia, qui sur le réseau Internet, par définition international, Flybase. Nous sommes bien, croyons-nous, dans l'ordre de la nature - si particulière soit-elle -, par définition coercitive. Et si culture il y a, ici, c'est celle de cette communauté qui regroupe les drosophilistes de par le monde. La spécificité du laboratoire japonais, pour un temps, semble être rendue caduque. II n'est qu'à considérer attentivement comment la drosophile est capable de passer d'un laboratoire (japonais) à un autre (français, par exemple) pour finir de s'en convaincre [17].

Les chercheurs, cependant, n'ont pas été réunis dans le but d'étudier les drosophiles en et pour elles-mêmes ni dans celui de vanter leurs mérites. Formant des agrégats de compétences diversifiées (neurobiologistes, biologistes moléculaires, comportementalistes, généticiens... ), ils constituent des dispositifs humains et techniques qui visent à manipuler les drosophiles. En ce sens, c'est aux fruits des manipulations - les mutants - qu'ils s'intéressent, bien plus qu'aux drosophiles «sauvages», les wild type. Le laboratoire japonais est

\footnotetext{
${ }^{6}$ Robert Kohler retrace le chemin ement qui a conduit la drosophile des poubelles au laboratoire et de l'organisme pis-aller à l'organisme de choix que nous connaissons aujourd'hui dans son livre, Lords of the fly [16]. II montre en particulier que les caracté ristiques chères aux drosophilistes $n$ 'ont pas toujours été tenues comme des qualités et que bien d'autres édé ments, notamment un environnement technique mais aussi «moral», sont entrés en ligne de compte dans cette constitution.
}

$\mathrm{m} / \mathrm{s} \mathrm{n}^{\circ} 12$, vol. 17, décembre 2001 ainsi un édifice fondé sur sept mutants sexuels - véritable cour des miracles où l'on trouve le mâle croaker qui met du temps avant de courtiser la femelle et produit une "chanson d'amour» (le bruit produit par les battements de ses ailes) particulière; les femelles spinster et chaste qui rejettent la copulation; les mâles fickle et okina chez lesquels le temps de copulation est modifié; le mâle lingerer qui tarde ou ne parvient pas à se désengager de sa partenaire; et le mâle satori qui rejette les femelles et montre de l'intérêt pour les autres mâles. A suivre ces miraculés, à analyser les processus par lesquels les chercheurs du laboratoire assurent leur existence au dehors, c'est une communauté de drosophilistes moins soudée en même temps qu'à une nature moins coercitive que nous découvrons.

\section{Le mal être des faits scientifiques: négocier l'existence des objets naturels}

Lorsque les mutants sexuels sont évoqués pour la première fois, nous sommes en 1988. Le directeur du laboratoire est alors directeur d'un projet de recherche qui se donne pour but de «comprendre les mécanismes moléculaires par lesquels les comportements coordonnés d'un organisme sont produits et modifiés d'une manière appropriée». La première phase du projet consiste à produire des lignées de drosophiles soumises à la mutagenèse et, au sein de celles-ci, à isoler celles qui ont trait au comportement. Le constat est positif: d'un stock de mille drosophiles «mutagénésées», le groupe obtient d'abord onze mutations, dont cinq qui montrent «un comportement de la reproduction défectueux». L'énoncé, tel qu'il apparaît dans les Progress Reports du laboratoire, a ici la platitude de ceux qui, construits a posteriori, ont effacé, pour les besoins de la présentation, les négociations qui les ont permises: I'équipe, visiblement, cherchait des mutants sexuels et elle en a trouvés. Pourtant, l'histoire de ces mutants montre qu'ils sont partiellement le fruit du hasard et ne constituent nullement le but que s'était fixé le directeur au moment d'entreprendre les recherches. II avait dans l'idée de s'in- téresser au problème de l'empreinte génomique mais «il était si difficile de suivre le comportement » des mutants obtenus dans ce schéma, qu'il se met en quête de "comportements innés plus stables, et c'était le comportement sexuel». L'objet «comportement sexuel » s'avère au final adéquat en ce qu'il est propre à répondre aux attentes du chercheur: trouver de nouveaux mutants et partant, ouvrir une voie de recherche originale susceptible de justifier de l'existence du laboratoire - et globalement, contribuer à asseoir le sens de l'innovation japonaise.

C'est lors de ce premier round que sont découvertes des mutations aux allures encore de matricule: 241201, 268907, 261206, 230501 deviennent bientôt, respectivement, croaker, spinster, lingerer et fickle. L'opération qui consiste à pourvoir ces nouveaux objets d'un nom qui ne soit pas un numéro est important, tant il est vrai qu'il en va des drosophiles comme des hommes: les nommer et ainsi les individualiser est constitutif de leur identité aussi sûrement que décrire la manière dont ils se comportent $[18]^{7}$. Toutefois, comme en attestent les épisodes suivants, cela ne suffit pas toujours à les faire venir à l'existence.

Les enjeux sur lesquels achoppe la nomination des mutants sont particulièrement visibles en ce qui concerne I'un d'eux, découvert lors du second pool génétique: satori. Le mutant est repéré par la caractéristique suivante: «Au début, nous pensions que ce mâle avait perdu le gène sexuel, et c'est pourquoi nous lui avons donné le nom de satori ». La formule est bien trouvée: satori est le terme japo-

\footnotetext{
L'anthropologue française Françoise Zonabend, parlant des hommes, écrit [18]: «Dans nos sociétés dites modernes, non moins que dans les sociétés dites archaïques, les anthroponymes ne sont jamais des termes interchangeables ou vides de sens. Ils rangent les personnes dans un système qui est en liaison directe avec d'autres systèmes de classification propres à la société en question, ou avec d'autres traits de la réalité sociale. IIs transmettent, du fait de leurs formes d'attribution, un message qu'il faut, selon les époques et les cultures, décoder. Enfin, ils ont une fonction d'identification: priver quelqu'un de son nom, le réduire à un matricule, c'est le rejeter dans le néant, hors de la communauté des hommes qui tous et partout portent un nom».
} 
nais pour désigner l'éveil au sens bouddhique du terme $[19]^{8}$. Les mâles satori sont distincts de leurs congénères parce qu'ils ne montrent aucun intérêt pour les femelles: libérés des désirs de ce monde, ils semblent bien avoir atteint l'éveil. Une étude plus approfondie montre cependant que si le mâle satori reste indifférent aux femelles, il est en revanche très attiré par les autres mâles. Les recherches permettent alors de conclure: «En résumé, satori est véritablement un mutant homosexuel » (figure 2). Éveillé, satori ne l'est donc pas tant que cela. II n'est finalement pas si détaché des plaisirs de ce monde; il les trouve ailleurs, c'est tout. Pour passer de satori digne représentant de la communauté bouddhique à satori homosexuel, il a nécessairement fallu procéder à quelques ajustements. Le nom de la mutation, cependant, ne compte pas parmi eux. S'il ne paraît plus approprié (il a perdu son référent), il est néanmoins conservé. Satori cesse peutêtre d'être "éveillé», il ne cesse pas d'appartenir au laboratoire japonais. Le nom fonctionne ici comme un sceau, reconnaissable de l'extérieur. Le processus dont il est question ici ne renvoie pas seulement à des problèmes d'identification et donc d'individualisation: il ne s'agit pas seulement de se décider pour l'asexualité ou l'homosexualité de satori. II renvoie également à des problèmes d'appartenance. La difficulté à faire admettre le nom de la mutation - et donc à la faire exister - en dehors du laboratoire et en dehors du Japon est, de ce point de vue, révélatrice. Un chercheur américain en visite dans le laboratoire japonais se demande ainsi pourquoi les scientifiques japonais continuent à appeler le mutant fruitless, satori. Satori, certes, ne correspond pas complètement à

\footnotetext{
${ }^{8}$ satori appartient au vocabulaire spécialisé par le quel, dans la littérature bouddhique, on désigne l'état de détachement du monde auquel on parvient au terme de pratiques religieuses ou ascétiques. Ainsi libéré de tous les liens humains, I'homme éveillé parvient à la connaissance de la vérité absolue. Pierre N akimovitch précise [19] : "L e bouddhisme pourrait se définir come culture de l'éveil: Sâkyamuni, son fondateur, n'est-il pas dénommé le buddha,
}

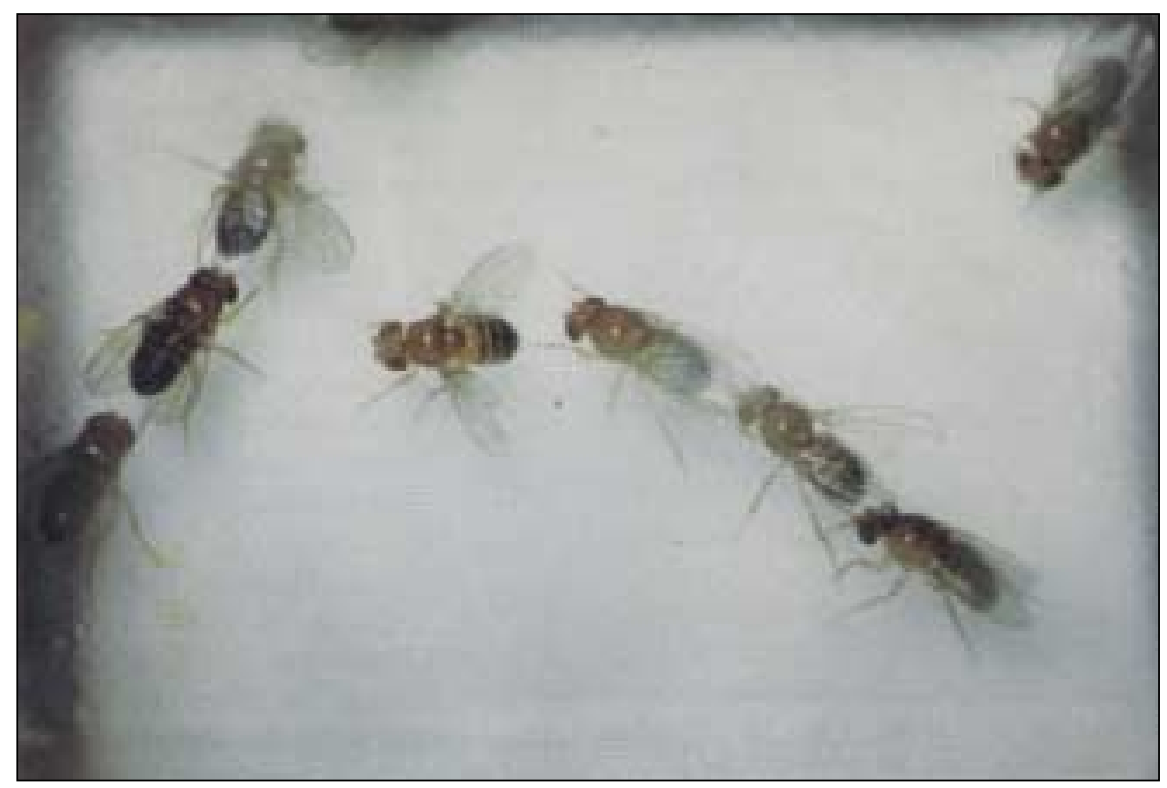

Figure 2. La parade «homosexuelle» des mutants mâles satori (cliché, Laboratoire Yamamoto).

fruitless, il est un allèle du gène fruitless. En général, m'explique-t-il, les chercheurs s'arrangent pour donner aux mutants des noms qui permettent de les connecter clairement avec le gène dont ils sont les allèles, comme fru ${ }^{1}$ ou fru ${ }^{2}$. Or, le mutant fruitless est attribué à une équipe de chercheurs américains et a vu le jour avant satori: la mutation a eu le temps de circuler sous ce nom et elle s'est même reproduite en plusieurs autres mutations, établies par le même groupe et nommées, comme il se doit, fru ${ }^{1}, f u^{2}, f r u^{3}$ et fru ${ }^{4}$. Nulle autre issue, pour le directeur du laboratoire japonais, que de reconnaître que son mutant satori est situé au même endroit de la carte génomique et, de ce fait, n'est qu'un mutant parmi d'autres dans l'ombre du mutant américain. Néanmoins, comme cherchant à me convaincre que la nuance génétique peut suffire à elle seule à pérenniser l'existence de satori en tant que mutation tout droit sortie de son laboratoire, il s'empresse de me montrer le schéma qui représente la séquence génétique sur laquelle se trouve satori. Dans la suite de l'histoire, c'est le phénotype (le comportement homosexuel) qui pourra sauver satori en continuant de le spécifier [17] ${ }^{9}$.
L'histoire de satori ne serait pas tellement ennuyeuse si elle était isolée. Pointant du doigt les problèmes rencontrés par un autre mutant, okina, le directeur du laboratoire japonais m'explique: «Ken est okina, c'est le nom de cette poupée sans sexe Le nom Ken, ce n'est pas nous qui l'avons trouvé, c'est une équipe américaine qui a isolé la même mutation... disons, une mutation différente sur le même gène, et à laquelle les chercheurs américains ont donné le nom de Ken and Barbie. Et notre nom, à nous, c'est okina. Mais ils ont publié avant». Comme pour ses autres mutants, le directeur japonais se montre fier de sa trouvaille: okina est un terme japonais ancien qui désigne un

\footnotetext{
${ }_{9}$ Dans l'article cité précédemment [17], je compare les points de vue du laboratoire japonais avec un autre, français, avec lequel il collabore, et en particulier les différends qui les opposent au sujet du comportement de satori. J'y analyse les éléments de ce qui ressemble fort à une controverse, le laboratoire français tenant I'homosexualité de satori comme n'allant pas de soi et avançant des arguments d'ordre technique (mettant en doute les dispositifs expérimentaux utilisés dans le laboratoire japonais) tout autant que social (se refusant à user d'une même terminologie pour parler des hommes et des mouches). Le destin de satori reste en suspend. L'indécision dont le mutant semble faire preuve n'est ce pendant pas à même de rompre la collaboration entre les deux laboratoires, laissant penser que la nature et son unité ne constitu ent pas le principal enjeu des pratiques scientifiques.
} 
vieillard portant une longue barbe blanche. Le mutant mâle, repéré par ses prouesses sexuelles pitoyables, tient son nom à l'âge avancé du bonhomme. Au mutant repéré par ces mêmes prouesses sexuelles pitoyables, l'équipe américaine choisit de donner le nom de ce couple de poupées en plastique - qui, effectivement, peuvent toujours essayer de faire des enfants... Avouons qu'il est bien dommage de devoir choisir entre les deux appellations, et le directeur japonais ne $s^{\prime} y$ résout pas. L'idée des existences multiples du mutant est séduisante: on imagine la même drosophile mener sa double vie, à ses heures et suivant les lieux, éphèbe impubère ou vieillard barbu...

Ces histoires laissent entrevoir une relation différente au fait scientifique. Dans la perspective du directeur japonais, okina peut fort bien cohabiter avec ken et satori avec fruitless sans que les uns portent ombrage aux autres. II ne se résout pas à l'uniformisation, ne se résout pas à choisir entre un mode d'existence et un autre. Les critiques du chercheur américain, non moins que les processus qu'elles dénoncent, sont culturellement marquées, elles aussi, aisément attribuables. D'où qu'ils viennent, les chercheurs occidentaux parlent toujours au nom de la science occidentale. Ils partagent les mêmes convictions, qui valent pour des vérités: la nature est coercitive et immuable - parce qu'elle est une et ordonnée. En ce sens, il est entendu qu'un seul et même objet naturel (un mutant, par exemple) ne peut avoir plusieurs existences. Une fois nommé, il est destiné à n'en mener qu'une seule: celle que lui a conférée son découvreur. Parce que les existences multipliées, dans l'ordre de la nature, sont proprement impensables: véritablement, ken est ken, fruitless est fruitless (définis chacun par les propriétés qui sont les leurs) - et le seront sinon de toute éternité, du moins jusqu'à ce qu'on vienne démontrer que l'énoncé était faux, ou incomplet. Ainsi, s'il advient que d'autres chercheurs proposent ailleurs, pour les mêmes objets, de nouvelles propriétés qui contredisent les premières, il faudra choisir et dé- terminer lesquelles des premières ou des secondes propriétés sont vraies; déterminer, du même coup, lesquels des premiers chercheurs ou des seconds ont raison.

\section{Le malêtre japonais: subir l'injustice naturelle}

Pourvoir les mutants d'une identité ne suffit pas toujours à les rendre solides ni à leur donner du poids à l'intérieur d'une communauté internationale qui compte des milliers de chercheurs, des centaines de laboratoires et d'innombrables autres mutations. Difficile de se faire une place. Dans chacune de ces négociations, c'est tout autant l'existence d'un objet naturel qui est sur la sellette que la pérennité du laboratoire et sa capacité à se faire reconnaître comme unité de recherche productive et autonome. Au final, l'enjeu dépasse de loin les difficultés techniques occasionnées par la proximité ou l'interaction de deux gènes ou allèles, les négociations renvoyant le directeur japonais à une situation qu'il ne connaît que trop bien et dont il fait régulièrement les frais: l'isolement du Japon par rapport au reste du monde.

Ici encore, c'est autour de satori que se cristallise la crise identitaire japonaise. Face au laboratoire japonais, le laboratoire américain dont est issue la mutation fruitless fait masse. Prenant concrètement la forme d'un consortium de laboratoires disséminés sur le sol américain, le groupe en question compte de nombreux scientifiques; à eux tous, m'explique le directeur, ils progressent vite parce qu'ils peuvent aisément communiquer les uns avec les autres; publient beaucoup parce qu'ils occupent des places stratégiques dans les revues importantes; menacent donc toujours un peu plus les avancées du laboratoire japonais. Les pouvoirs sont ainsi naturellement déséquilibrés et empêchent une quelconque manifestation de confiance ou forme de collaboration. L'isolement insulaire, à lui seul, retient d'entretenir avec les laboratoires américains des «relations régulières», qui permettraient que chaque groupe publie à son heu- re sans empiéter sur la publication du groupe voisin - qui feraient, en somme, que les laboratoires japonais et américains avancent de concert et non de front.

Dans la course à la publication que mènent les deux groupes, le laboratoire japonais sort pour une fois vainqueur: l'équipe japonaise publie avant l'équipe américaine un article dans le journal Proceedings of $\mathrm{N}$ ational Academy of Science, sur le mutant satori [20]. L'article américain ne paraît que quelques mois plus tard dans le journal Cell. Le laboratoire japonais, cependant, ne savoure sa victoire que peu de temps. Dans leur article, les chercheurs américains se font la part belle, leurs coéquipiers japonais n'étant mentionnés que pour avoir cloné une «partie du gène»: "H ere we report the cloning and characterization of the fru gene through two convergent lines of research. (...) At the time this paper was about to be submitted, a paper appeared that reported cloning part of the fru gene» [21]. Comble de l'injustice: c'est l'article américain qui est retenu et célébré dans l'éminente revue Science [22]. Le travail du groupe américain y est salué comme "une véritable percée» tandis que celui de l'équipe japonaise y est à peine évoqué. Le directeur du laboratoire japonais est dépité par la nouvelle: "Ouais... "partie du gène"... Ça veut dire travail partiel !». D'autres chercheurs du laboratoire, à leur tour, s'étonnent de cette attribution mesquine: "Qu'est-ce que ça veut dire, partiellement ? $10 \%$ ? $99 \%$ ? Ce n'est pas vraiment scientifique». Les membres du laboratoire japonais ne sont pas dupes: I'éloignement, I'isolement sur l'archipel nippon ne sont pas seuls en cause dans cette affaire. Des chercheurs venus d'ailleurs viennent mettre en doute la légitimité de l'existence des objets qu'ils produisent: satori-le-Japonais est fruitlessl'Américain, okina-le-Japonais est kenl'Américain. "Tous les mutants du laboratoire japonais existaient déjà avant», "les chercheurs japonais n'ont rien inventé», s'entendent dire les chercheurs japonais. Le couperet culturel tombe une nouvelle fois, alors même que les chercheurs japonais se croyaient en terrain neutre - dans 
l'antre même de l'internationalisme. Et les mêmes discours reviennent comme une litanie: les Japonais sont imitateurs (sont de bons imitateurs, veut la formulation positive), ils ne sont pas innovateurs (sont de piètres innovateurs, veut le pendant à la formulation positive). L'histoire est donc toujours la même: les chercheurs du laboratoire japonais, comme tous les scientifiques japonais, puisent dans le lot d'objets déjà constitués (ken, fruitless), les transforment (en vieillard barbu, en homosexuel), et se les approprient (okina, satori). Ces histoires, c'est évident, montrent donc qu'il ne suffit pas d'être scientifique pour entrer de plain pied dans l'internationalisme qui, dit-on, caractérise l'activité scientifique. Tous ne sont pas égaux en la matière; et l'accès aux réseaux sociaux qui font les publications n'est pas le même pour tous. La communauté des drosophilistes semblait une occasion trop belle pour le laboratoire japonais de se dégager du pesant fardeau culturel et voilà que les processus d'identification et de nomination des mutants, pernicieusement, le réintroduisent.

\section{À circonstances particulières, solutions spécifiques: créer du lien social}

Tout ce que nous avons vu jusqu'à maintenant conduit à penser qu'il est tout aussi important de s'assurer de la fiabilité des produits destinés à sortir du laboratoire que de garantir au laboratoire des partenaires solides et dispersés - constituant autant de points d'appui permettant au laboratoire japonais de faire, à la force du poignet, ce que les laboratoires américains font «naturellement»: communiquer, créer un réseau et le faire fructifier. Les outils développés par le laboratoire japonais n'ont pas seulement trait à la spécification des objets naturels qu'il produit; ce sont également des outils sociaux, rhétoriques, stratégiques, qui visent à faire rentrer ces mêmes objets dans le lot des faits scientifiques.

Considérons ce qui arrive au mutant fickle. Une première version d'un ar- journal Cell. Les auteurs reçoivent rapidement l'indication que l'article pourra être accepté pour publication, sous réserve cependant de quelques modifications. Au nombre de celles-ci, le laboratoire japonais doit renoncer à l'appellation fickle et rendre à César ce qui lui appartient: le gène a en effet été découvert par une autre équipe qui l'a nommé Dsrc29A (peut-on se permettre de dire qu'on préférait le plus éloquent fickle ?) et les données apportées par l'équipe japonaise ne sont pas jugées suffisantes pour renommer le gène. II est presque question de droits d'auteur ici. Dans la seconde version de l'article, le directeur japonais se livre à un petit tour de passe-passe qui lui permet de répondre à la demande de l'arbitre tout en préservant I'appellation de son cru: "We use the name Dsrc29A for this gene and fickle is used as the name of a mutation in the Dsrc29A gene». Passant de nom de gène à nom de mutation, fickle est malgré tout sauvé, et le laboratoire japonais avec: dorénavant, Dsrc29A devra compter avec fickle et le laboratoire-mère avec le laboratoire japonais. Ainsi est tissé le fil d'un réseau invisible qui réfère le travail d'un laboratoire à un autre, I'un menant nécessairement à l'autre. L'équipe japonaise assure, en ce point, son existence au sein de la communauté internationale.

Mais I'histoire ne s'arrête pas là. Dans le premier article, fickle est présenté comme un gène ayant pour «site d'action » le cerveau. Dans cette première vie de fickle, il est ainsi établi un lien entre un gène, une «machinerie neuronale» et le comportement sexuel. Nulle trace «d'anormalités dans les muscles ni dans l'appareil génital » de l'insecte, affirment les chercheurs japonais. Les arbitres, cependant, incitent à la prudence et à de nouveaux contrôles: les données produites par le laboratoire japonais ne sont pas à même de produire une assertion qui soit tenue pour vraie. Dans leurs commentaires, les arbitres semblent tenir pour acquis que les scientifiques ne peuvent parler en leur nom au travers «d'observations non publiées»: ce sont les faits qui doivent parler et les données expérimentales doivent venir à la rescousse des chercheurs. Le processus expérimental est donc relancé et avec lui l'identité potentielle du mutant. Le directeur du laboratoire japonais explique: "Je pense que l'article sur fickle était presque accepté, tous les reviewers semblaient assez excités et l'éditeur avait même menti on né le nombre de caractères que l'article devait contenir. Mais en réponse à I'un des arbitres qui demandait d'autres expé riences, nous avons fait une nouvelle observation : un défaut dans l'appareil génital ! (... ) M aintenant, c'est la conception entière du papier qui est à revoir et la nouvelle version concernera le développement génital, et non pas la génétique du comportement ! On doit donc changer quelques figures, etc., et le con texte général sera différent. L'article doit être réécrit commesi on avait eu l'intention d'étudier le disque génital. Le manuscrit est prêt mais comme nous ne sommes pas habitués à travailler sur le disque génital, nous avons besoin de faire quelques expériences d'abord et on se bat pour obtenir de bons clichés et des données à inclure dans la nouvelle version ». La réécriture de l'article, qui est ici le fait du directeur du laboratoire et non des chercheurs conduisant les recherches, est concomitante de l'expérimentation. La découverte d'une malformation de l'appareil génital de fickle amène à modifier I'article. II faut changer les figures, changer le contexte. Et au bout de tout cela, sort un nouvel article qui aura comme nouveau point d'entrée: «le développement génital ». Ce n'est pas ici fickle qui, au départ, change de nature (le directeur ne parle pas d'un nouveau fickle), c'est l'article qui change de forme et partant, fickle qui change finalement de nature. Dans la nouvelle version, fickle s'appelle Dsrc29A et il est une mutation qui affecte non pas une partie du système nerveux central mais l'appareil génital. Dans cet exemple, ce sont l'écriture et la publication qui imposent aux objets naturels des contraintes propres, différentes de celles de l'expérimentation. Elles constituent en même temps des outils prenant part à leur formation.

Tout au long de cette chaîne de négociations, le directeur du laboratoire japonais ne fait nulle mention de la vérité de fickle. Plutôt que d'être te- 
nus soit pour vrais, soit pour faux, les faits naturels sont organisés comme une histoire dont les tenants peuvent être changés. La nouvelle histoire présente de nouveaux personnages avec de nouveaux rôles - et ceux-là, comme ceux de l'histoire précédente, constituent une structure cohérente. Les arguments du directeur ne viennent donc pas porter préjudice aux données produites par ses membres concernant la localisation neuronale de fickle. Ces données constituent au contraire les points d'accroche d'une autre vie de fickle tout autant que les nouvelles données initient une vie à venir, enrôlant le mutant dans un autre système. Dans ce nouveau système, c'est tant le mutant lui-même qui sera amené à côtoyer d'autres partenaires ( $d$ 'autres gènes avec lesquels il interagira) que les membres du laboratoire qui seront destinés à se trouver d'autres alliés (d'autres laboratoires avec lesquels collaborer, d'autres avec lesquels se battre).

La délocalisation à laquelle est soumise la pratique scientifique dans l'idéologie officielle rend donc mal justice aux efforts fournis localement pour la spécifier. Dans le laboratoire japonais étudié, les pratiques se donnent à voir comme locales, tout autant que la nature. Prenant le contrepied de la formulation utilisée par le chercheur américain, le directeur du laboratoire japonais a les moyens de dire qu'ici, dans ce laboratoire installé au Japon, ken est okina et fruitless est satori - ou plus justement encore, ken est à okina et fruitless est à satori ce que le laboratoire américain est au laboratoire japonais: le fruit de configurations toujours localisées. Partant de cette dimension locale dont, finalement, il n'est peut-être pas nécessaire de s'extraire, le laboratoire japonais propose ni plus ni moins qu'une nouvelle manière d'envisager les faits scientifiques: là, les objets naturels sont laissés en leur état d'indétermination qui rend possible des existences multiples. Rendant visibles les rouages sociaux qui font la pratique scientifique, les propos du directeur du laboratoire japonais déroutent plus d'une fois par une $\mathrm{m} / \mathrm{s} \mathrm{n}^{\circ} 12$, vol. 17, décembre 2001 transparence aveuglante. Affirmer comme il le fait que la pratique scientifique dépend de la maîtrise de réseaux sociaux, affirmer qu'elle met en œuvre des stratégies (de composition, de publication, etc.), tend à faire de lui un sociologue des sciences. Il aurait pu être l'auteur d'une phrase que l'on doit au sociologue Michel Callon: "Pris dans la tourmente créatrice de nouveaux réseaux ou en châssés dans la logique conservatrice des anciens, les faits scientifiques sont toujours impurs par nature, voire par destination. IIs sont faits de bric et de broc; ils sont composés d'éléments hétérogènes, associant des compétences à des équipements, des textes à des savoirs tacites, des humains à des non humains. C'est de cette impureté que dé pend leur capacité à résister et à intéresser» [23]. Le directeur et son laboratoire illustrent l'efficacité des catégories analytiques produites par les sociologues des sciences - mieux, ils les déploient. Ils produisent une sociologie des sciences indigène qui s'oppose nettement à une épistémologie exogène, proposant une épistémologie enracinée dans le social et non pas dans un rapport idéel à la nature.

\section{Conclusions}

Le laboratoire japonais, en même temps qu'il fait valoir ce qui le relie à la communauté scientifique internationale, n'abdique donc pas pour autant l'ordre des spécificités locales: avec le double effet de faire saisir les conditions particulières dans lesquelles s'effectue la recherche au Japon - de rendre du même coup ses avancées spectaculaires -, et de restituer à la cosmologie occidentale moderne sa juste dimension. Fonctionnant suivant des schèmes qui leur sont propres et dans lesquels les hommes et les objets dits naturels ont un mode d'existence spécifique, les deux systèmes sont tout aussi locaux I'un que I'autre. L'un a simplement l'avantage sur l'autre d'avoir une plus longue histoire. Comme la cosmologie moderne occidentale, la cosmologie japonaise aspire à l'universalité - aspire à s'étendre du local au global - mais avec d'autres moyens: en s'appuyant non pas sur les forces d'une nature dans laquelle on peine à reconnaître une unité mais sur les forces humaines qui font la trame de la société

\section{RÉFÉRENCES}

1. Lenclud G. Le grand partage ou la tentation ethnologique. In: Althabe G, Fabre D, eds. Vers une ethnologie du présent. Collection Ethnologie de la France. Paris: Editions de la Maison des Sciences de l'H omme, 1992: 9-37.

2. Lévi-Strauss C. La pensée sauvage. Paris: Pion, 1962.

3. Serres M. Le contrat naturel. Paris: Flammarion, 1992 (1990) : 127.

4. Kuhn T. L a structure des révolutions scientifiques. Paris: Flammarion, 1983 (1962).

5. Lynch M. Technical work and critical inquiry: Investigation s in a scientific laboratory. Social Studies of Science 1982; 12: 499-533.

6. Lynch M. Scientific practice and ordinary action. Ethnomethodology and social studies of science. Cambridge: Cambridge University Press, 1993.

7. Traweek S. Beamtimes and lifetimes. The world of high energy physicists. Cambridge, Massachusetts, London: H arvard University Press, 1992 (1988).

8. Latour B, Woolgar S. La vie de laboratoire. La production des faits scientifiques. Paris: La Découverte, 1993.

9. Vinck D. Sociologie des sciences. Paris: Armand Colin, 1995: 267.

10. Shapin S, Schaffer S. Léviathan e la pompe à air. $H$ obbes et Boyle entre science ed politique. Paris: La Découverte, 1993 (1985).

11. Houdart S. Ce que l'ethnographie d'un laboratoire scientifique japonais nous apprend sur le sens de la différence au Japon. In: Gossot A, Berthon JP, eds. Japon plurid 3. Arles: Philippe Picquier, 1999: 83-91.

12. Sugimoto $M$, Swain D. Science and culture in traditional Japan. Rutland, Tokyo: Charles E. Tuttle Company, 1989.

13. Low FL. The butterfly and the frigate: social studies on science in Japan. Social Studies of Science 1989; 19: 313-42.

14. Natsume $S$. La civilisation japonaise moderne (Gendai Nihon no kaika, 1911). In: Allioux AM ed. Cent ans de pensée au lapon (1). Arles: Philippe Picquier, 1996: 127-56.

15. Knorr-Cetina K. Laboratory studies. The cultural approach to the study of science. In : Jasanoff S, Markle GE, Petersen JC, Pinch P, eds. Handbook of science and technology studies. Thousand Oaks, London, New Delstudies. Thousand O aks, London, New
hi : SAGE Publications, 1995: 140-66.

16. Kohler R. Lords of the fly. Drosophila genetics and the experimental life. Chicago, London : The University of Chicago Press, 1994.

17. Houdart S. Quand les mouches font la différence. D'un laboratoire (japonais) à I'autre (français). Ateliers 2000; 19: 67-94. 


\section{RÉFÉRENCES}

18. Zonabend $F$. Le nom de personne. L'H omme 1980; 20: 7-23.

19. Nakimovitch $P$. Pensées et écrits de Dôgen (1200-1253). Ébisu 2000; 24: 17.

20. Ito $\mathrm{H}$, et al. Sexual orientation in Drosophila is altered by the satori mutation in the sexdetermination gene fruitless that encodes a zinc finger protein with a BTB domain. Proc Natl Acad ScI USA 1996; 93: 9687-92.

21. Ryner LC, et al. Control of male sexual behavior and sexual orientation in Drosophila by the fruitless gene. Cell 1996; 87: 1079-89.

22. Fly sex drive traced to fru gene. Science 1996 ; $274: 1836$.

23. Callon $M$. La science et ses réseaux. Genèse et circulation des faits scientifiques. Paris: La Découverte/ Conseil de l'Europe/ Unesco, 1989: 32.

\section{Sophie Houdart}

Japanese Society for the Promotion of Science, Flat Ohtake I.102, Toshima-ku Takada 2-18-28, 171.0033 Tokyo, Japon.

E-mail : Shoudart@aol.com

\section{Summary}

From flies and humans: behavioral genetics in a Japanese laboratory

Anthropology of science allows to consider scientific practices as well as scientific statements from the point of view of their social and cultural context of elaboration. In this respect, the ethnographic study of a Japanese laboratory of behavioral genetics provides data on the articulation of local dimension (a laboratory in Japan) and global dimension (the international scientific community). In this paper, the author analyses some of the processes through which researchers give birth to scientific facts. It shows finally how the Japanese laboratory constitutes a place where people own specific means of expression and action to extend outside the wall of the laboratory new cultural configurations.

\section{TIRÉS À PART}

\section{S. Houdart}

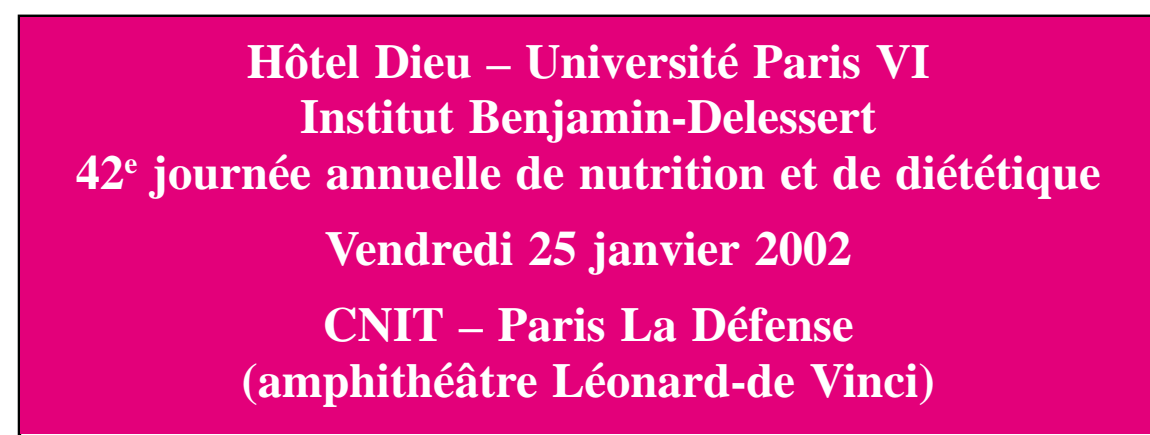

Président : professeur Bernard GUY-GRAND

Vice-Présidents : professeur Arnaud BASDEVANT, professeur Bernard MESSING, professeur Gérard SLAMA

Secrétaire générale: Marie-France CARRIÉ-MOYAL

Fondateurs : professeur Henri BOUR, professeur Maurice DÉROT, docteur Guy HÉRAUD

Membre d'honneur: docteur Michel RATHERY

\section{MATINÉE}

\section{Plantes : alimentation et santé}

\section{Modérateurs : Professeur Arnaud BASDEVANT} Professeur Bernard BEAUFRÈRE

9h 15 Allégations santé : du complément alimentaire au médicament Pr Bernard BEAUFRÈRE (Clermont-Ferrand)

9h45 Les phyto-nutriments Pr Robert ANTON (Strasbourg)

$10 \mathrm{~h} 45$ Les phyto-stérols Pr Philippe MOULIN (Lyon)

$11 \mathrm{~h} 15$ Les phyto-œstrogènes Pr Jacques BRINGER (Montpellier)

$11 \mathrm{~h} 45$ Conclusions

12 h 00 Remise du Prix Benjamin-DELESSERT 2002

et Lecture Benjamin Delessert:

Promesses et limites des microconstituants des végétaux

dans le domaine des relations alimentation/santé

Dr Gérard PASCAL (Paris)

\section{APRÈS-MIDI}

Activité physique, nutrition et santé

Modérateurs : Professeur Bernard GUY-GRAND

\section{Professeur Bernard MESSING}

$14 \mathrm{~h} 30$ Activité physique et maladies de la nutrition : les enjeux

$$
\text { Dr Jean-Michel OPPERT (Paris) }
$$

$15 \mathrm{~h} 00$ Les effets physiologiques de l'activité physique

Pr Charles-Yannick GUEZENNEC (Brétigny-sur-Orge) 15 h 30 Comment évaluer l'activté physique ?

16 h 00 Que conseiller en pratique médicale?

Dr Chantal SIMON (Strasbourg)

Dr Jean-François GAUTIER (Paris) 